\title{
Vaginal relapse after laparoscopic hysterectomy in early endometrial carcinoma: does the intrauterine manipulator affect the results?
}

\author{
Santiago Domingo • Alfredo Perales-Puchalt • \\ Jose María Vila-Vives • Marta Gurrea • Antonio Pellicer
}

Received: 5 December 2011 / Accepted: 12 January 2012 /Published online: 31 January 2012

(C) Springer-Verlag 2012

\section{Introduction}

Laparoscopic approach for the treatment of early endometrial carcinoma is currently performed in many institutions. This approach has successfully been compared with laparotomy in the surgical staging and treatment of endometrial carcinoma [1]. The FIGO guidelines for anatomical-surgical staging of endometrial carcinoma can be carried out by laparoscopic approach [2]. The possibility of laparoscopic assistance for pelvic lymphadenectomy allows a non-invasive surgery for this prevalent malignancy, with all the known advantages.

With the advent of the laparoscopic hysterectomy for the treatment of endometrial carcinoma, some questions have been raised. First, the possibility of malignant peritoneal dissemination. No differences have been reported between laparotomy and laparoscopy with regard to the incidence of positive peritoneal cytology after surgery [3]. Second, the risk of laparoscopic port-site metastases, which have been described in almost all types of gynecological cancers [4]. Third, the risk of vaginal cuff recurrence, which generates controversy about the use of intrauterine manipulators [5].

We report the case of a patient with a non-invasive endometrial carcinoma treated with total laparoscopic hysterectomy (TLH) and pelvic lymphadenectomy using an intrauterine manipulator, who showed a vaginal cuff recurrence 11 months later.

S. Domingo $\cdot$ A. Perales-Puchalt $(\bowtie) \cdot J$. M. Vila-Vives $\cdot$

M. Gurrea $\cdot$ A. Pellicer

Department of Obstetrics and Gynaecology,

Hospital Universitario La Fe,

Bulevar Sur, s/n,

46026 Valencia, Spain

e-mail: alfredoperales@comv.es

\section{Case report}

A 56-year-old menopausal woman presented to her physician with complaints of vaginal bleeding. She had no history of hypertension or diabetes mellitus, and had been using hormonal replacement therapy for 5 years. The ultrasound scan showed a regular endometrial cavity of $10 \mathrm{~mm}$, without signs of deep invasion. An endometrial curettage was performed, reporting an endometrioid adenocarcinoma grade 1 . Two weeks later, consequently with the findings of the biopsy, she underwent a TLH with bilateral salpingooophorectomy and bilateral pelvic lymph node sampling. An intrauterine manipulator was used to assist the hysterectomy (V-CARE standard; ConMed ${ }^{\circledR}$ Corporation, Utica, NY). Prior to the hysterectomy we sealed both fallopian tubes with bipolar coagulation and performed a pelvic washing for posterior pathological study. We coagulated and transected the round ligament, dissected laterally the peritoneum from the infundibulopelvic ligament and identified the ureter and uterine artery. The bladder was also dissected away up to the vagina. Both infundibulopelvic and uterine artery were coagulated and transected. The vagina was open using a monopolar cutting, following the top of the manipulator cup. Finally, the uterus was removed vaginally, and the vagina sutured using intracorporial knots. Histological evaluation revealed an endometrial benign polyp, a leiomyoma, and an atrophic endometrial mucosa. Cervix, fallopian tubes, and ovaries were normal. Nine analyzed lymph nodes were negative for malignancy, and the pelvic washing was negative for malignant cells. Final pathologic diagnosis was a stage IA FIGO endometrial carcinoma grade 1 and no further treatment was given.

During the follow-up at 6 months from surgery, the patient was free of malignancy (cytology of the vaginal cuff and pelvic examination). Eleven months after surgery, 
routine follow-up examination revealed a $12 \times 19 \times 13 \mathrm{~mm}$ asymptomatic nodule in the vaginal cuff, which was confirmed by pelvic ultrasound. Fine needle aspiration of the tumor was positive for malignant cells and the biopsy confirmed a recurrent adenocarcinoma similar to the patient's primary endometrial tumor. A magnetic resonance showed a 3 -cm nodule in the vaginal cuff adherent to the posterior wall of the bladder, suspected for recurrence of endometrial cancer. Cystoscopy and colonoscopy showed no signs of malignancy.

We performed a laparotomic resection of the vaginal cuff. Pathology showed that the resected mass had the same histology as the endometrial carcinoma previously treated, infiltrating the mucosa and muscular vagina. The lymphovascular space was also involved, and the superior surgical margin was affected by the tumor, while the deep surgical margin was tumor-free. The result of the peritoneal cytology was negative for tumor spread. Given the aggressiveness of the recurrent disease, the original pathology was reviewed, but no changes were made in the diagnosis. The patient was referred for radiation therapy and received a course of external beam pelvic radiotherapy (total radiation $50 \mathrm{~Gy}$ ) followed by inpatient vaginal cylinder placement.

Cytology of the vaginal cuff, chest X-ray, abdominopelvic CT, and tumor markers showed no evidence of residual or new disease in the follow-up. The tumor recurred again 40 months after the second surgery, and the patient died due to a deep venous thrombosis.

\section{Discussion}

Laparoscopic approach in endometrial carcinoma is performed in many institutions as the elective surgical procedure for early stages. Retrospective and prospective studies have evaluated the validity of laparoscopic surgery in patients with endometrial cancer, with no different longterm outcome when compared to laparotomic approach and a better quality of life in the recovery period $[6,7]$. The prospective randomized trial conducted by Malur et al. compared the laparoscopic-assisted vaginal hysterectomy (LAVH) approach with the total abdominal hysterectomy (TAH) for the treatment of patients with endometrial carcinoma FIGO stages I-III [8]. The authors reported that the LAVH is associated with lower perioperative morbidity compared with the TAH, therefore it can be recommended. However, they consider that the number of enrolled patients was too small and that the followup was too short. A randomized trial that compared laparoscopy $(n=1,696)$ versus laparotomy $(n=920)$ for patients with endometrial carcinoma stage I to IIA concluded that laparoscopic approach is feasible and also safe in terms of short-term outcomes[9].
Although rare, one of the major concerns of laparoscopy in gynecologic cancer is the development of port-site and vaginal cuff metastasis, which have been described even in early stages and after brachytherapy $[4,5]$. The mechanism that may explain how the malignant cells reach and implant in the wound are still unclear. The most plausible hypotheses are the dissemination of malignant cells through the fallopian tubes to the abdominal cavity, the direct contact of lymphatic nodes with the wound and the contamination of endometrial material through the cervical ostium. To avoid port-site metastases in laparoscopic surgery, several procedures are used although their effectiveness is still not proved. These procedures are: washing the wounds after the surgery, trocar fixation to avoid its way out, ascites suction, and trocar site closure; fallopian tube sealing as first step in the surgery and extraction of the lymph nodes in a bag [10]. No clear recommendations have been reported to avoid the vaginal cuff relapses. In order to prevent it, surgeons historically employed cervical occlusion techniques, such as preoperative suture closure of the cervix [11].

Most series in the literature do not describe whether they use an intrauterine manipulator in their surgeries or not. This instrument, when used in cases of endometrial carcinoma, may help the peritoneal and vaginal dissemination. Our case had a stage IA, grade I carcinoma, and no malignant tissue was supposed to be in the endometrial cavity, because it was completely removed during the curettage.

Another way intrauterine manipulator can help malignant cell dissemination to peritoneal cavity is by uterine perforation, particularly in cases with deep myometrial invasion.

The use of an intrauterine manipulator is not compulsory in laparoscopic hysterectomy [5], and although this instrument is very helpful during surgery, TLH should be performed without it in endometrial carcinoma to avoid recurrences until proper prospective studies reassure its safety.

\section{Conclusion}

This case report supports the need of prospective and randomized trials to study the higher risk of vaginal relapse with the use of intrauterine manipulators in the laparoscopic management of endometrial carcinoma. Meanwhile, we must have some consideration for the use of intrauterine manipulators in the laparoscopic management of endometrial carcinoma because, although not proved, their use could increase the risk of vaginal relapse.

Declaration of interest The authors confirm no conflicts of interest. The authors alone are responsible for the content and writing of the paper. 


\section{References}

1. Mourits MJ, Bijen CB, Arts HJ, ter Brugge HG, van der Sijde R, Paulsen L, Wijma J, Bongers MY, Post WJ, van der Zee AG, de Bock GH (2010) Safety of laparoscopy versus laparotomy in earlystage endometrial cancer: a randomised trial. Lancet Oncol 11:763-771

2. Frey MK, Ihnow SB, Worley MJ Jr, Heyman KP, Kessler R, Slomovitz BM, Holcomb KM (2011) Minimally invasive staging of endometrial cancer is feasible and safe in elderly women. $\mathrm{J}$ Minim Invasive Gynecol 18:200-204

3. Eltabbakh GH, Mount SL (2006) Laparoscopic surgery does not increase the positive peritoneal cytology among women with endometrial carcinoma. Gynecol Oncol 100:361-364

4. Muntz HG, Goff BA, Madsen BL, Yon JL (1999) Port-site recurrence after laparoscopic surgery for endometrial carcinoma. Obstet Gynecol 93:807-809

5. Chu CS, Randall TC, Bandera CA, Rubin SC (2003) Vaginal cuff recurrence of endometrial cancer treated by laparoscopic-assisted vaginal hysterectomy. Gynecol Oncol 88:62-65
6. Magrina JF, Weaver AL (2004) Laparoscopic treatment of endometrial cancer: five-year recurrence and survival rates. Eur J Gynaecol Oncol 25:439-441

7. Kluivers KB, Ten Cate FA, Bongers MY, Brolmann HA, Hendriks JC (2011) Total laparoscopic hysterectomy versus total abdominal hysterectomy with bilateral salpingo-oophorectomy for endometrial carcinoma: a randomised controlled trial with 5-year follow-up. Gynecol Surg 8:427-434

8. Malur S, Possover M, Michels W, Schneider A (2001) Laparoscopic-assisted vaginal versus abdominal surgery in patients with endometrial cancer - a prospective randomized trial. Gynecol Oncol 80:239-244

9. Walker JL, Piedmonte MR, Spirtos NM, Eisenkop SM, Schlaerth JB, Mannel RS, Spiegel G, Barakat R, Pearl ML, Sharma SK (2009) Laparoscopy compared with laparotomy for comprehensive surgical staging of uterine cancer: gynecologic Oncology Group Study LAP2. J Clin Oncol 27:5331-5336

10. Ramirez PT, Wolf JK, Levenback C (2003) Laparoscopic port-site metastases: etiology and prevention. Gynecol Oncol 91:179-189

11. Pratt JH, Symmonds RE, Welch JS (1964) Vaginal hysterectomy for carcinoma of the fundus. Am J Obstet Gynecol 88:1063-1071 